\title{
Population migration, confirmed COVID-19 cases, pandemic prevention, and control: evidence and experiences from China
}

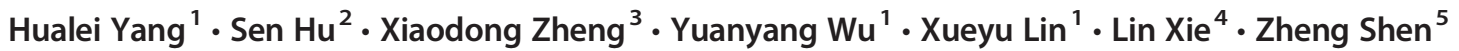

Received: 2 May 2020 / Accepted: 15 October 2020 / Published online: 24 October 2020

(C) Springer-Verlag GmbH Germany, part of Springer Nature 2020

\begin{abstract}
Aim The virulence of the novel coronavirus disease (COVID-19) has facilitated its rapid transition towards becoming a pandemic. Hence, this study aims to investigate the association between population migration and the number of confirmed COVID19 cases in China while investigating its measures for pandemic prevention and control.

Subject and methods A susceptible-exposed-infected-recovered-dormancy (SEIRD) model for the spread of COVID-19 in China was created to theoretically simulate the relationship between the populations migrating from Wuhan and the number of confirmed cases. Data from Baidu's real-time dynamic pandemic monitoring system were elicited to empirically examine the theoretical inferences.

Results Populations migrating from Wuhan to other cities increased the initial number of latently infected cases in these cities, raising the number of confirmed cases. Hence, implementing social distancing between the susceptible and infected populations could effectively lower the number of infected cases. Using data from Baidu's real-time dynamic pandemic monitoring system, the empirical results revealed that an increase of 1000 persons migrating from Wuhan raised the number of confirmed cases by 4.82 persons.

Conclusion This study confirmed the positive association between population migration and the number of confirmed COVID19 cases. Based on the theoretical and empirical analysis, China's pandemic prevention and control measures are discussed.
\end{abstract}

Keywords Wuhan $\cdot$ Novel coronavirus disease $\cdot$ SEIRD model $\cdot$ COVID-19 $\cdot$ Prevention and control $\cdot$ Pandemic

\section{Introduction}

In December 2019, three patients with cases of pneumonia, originating from an unknown cause, emerged from Wuhan

Xiaodong Zheng

zhengxd@zjgsu.edu.cn

1 School of Public Administration, Zhongnan University of Economics and Law, Wuhan 430073, China

2 School of Management Science and Engineering, Nanjing University of Information Science and Technology, Nanjing 210044, China

3 School of Economics, Zhejiang Gongshang University, No.18 Xuezheng Street, Xiasha New Higher Education Park, Hangzhou 310018, Zhejiang, People's Republic of China

4 Institution of Population and Labor Economics, The Chinese Academy of Social Science, Beijing 100028, China

5 School of Economics and Management, Zhejiang A\&F University, Hangzhou 311300, China
City's Huanan Seafood Market. They were admitted to Wuhan Jinyintan Hospital for treatment and all three patients were all diagnosed with the novel coronavirus disease (COVID-19). Since then, COVID-19 has gained public attention (Huang et al. 2020a, b; Guan et al. 2020; Li et al. 2020; Wang and Jin 2020). As populations heavily migrated in China due to the New Year's festivities, COVID-19 spread rapidly and opportunistically. As of June 18, the number of confirmed cases in China had exceeded 84,000, and the number of mortalities had exceeded 4600 yielding a fatality rate of $5.47 \%$. Specifically, there were more than 50,000 confirmed cases from Wuhan, where the case fatality rate was as high as $7.69 \%$.

According to epidemiologic theory, a combination of susceptible and infected populations, together with transmission agents, determines the number of people infected by a pandemic. Specifically, the migration of latently infected and infected populations catalyse a pandemic's spread. To mitigate the pandemic's damage to the economy and society, the Chinese government implemented joint prevention and 
control strategies, issuing labour and production shutdowns, and imposing a strict lockdown on all urban and rural communities (Chen et al. 2020a, b; Gong et al. 2020; Deng and Peng 2020). The efficacy of the intervention eventually allowed China to attain control over the pandemic. To effectively respond to similar public health emergencies in the future, China needs to summarize patterns and experiences pertaining to the pandemic (Zhang 2020; Xu et al. 2020). Notably, these stratagems should account for the mechanism of action associated with population migration according to the spread of the pandemic. Hence, China's experiences concerning the prevention and control of COVID-19, based on the variable of population migration, are of the utmost importance.

China's battle against the pandemic is nearing an end; however, due to globalization and expeditious migrations, various countries are gradually falling prey to the pandemic. As of June 18, COVID-19 cases had appeared in over 120 countries. Outside of China, there have been more than 8,300,000 confirmed cases with over 400,000 mortalities, yielding a case fatality rate of $5.3 \%$. Among them, there have been more than 300,000 confirmed cases and more than 40,000 mortalities in the UK, which reports a fatality rate of $14 \%$, while more than 230,000 confirmed cases and 30,000 mortalities have been reported in Italy with a fatality rate as high as $14.5 \%$. Facing such a severe pandemic, the United States entered into a state of 'national emergency'. Many high-ranking officials of the United Kingdom were also infected, and countries such as Italy and Spain declared nation-wide lockdowns and issued travel bans.

Despite continuous advancement in measures of prevention and control, the virulent trajectory of the global pandemic is far from being curbed. Due to China's effective prevention and control of the pandemic, the World Health Organization's Director-General, Tedros Adhanom, advocated that each country should learn from China's experience (Zhang et al. 2020). The Chinese government's organization and mobilization capabilities - especially stratagems for intercepting population migration, such as city-wide lockdowns - have garnered the attention of experts from across the world (Prem et al. 2020; Li et al. 2020; Kucharski et al. 2020; Tian et al. 2020).

This study aims to answer two questions. First, we attempt to investigate how population migration affects the number of confirmed cases in each region, and summarize the pandemic's patterns of transmission. Second, based on our theoretical and empirical analysis of population migration's impact on the number of confirmed cases, we will provide an overview of China's experiences with early warning, prevention, and control of the spread of the pandemic, from the perspective of intercepting population migrations.

The structure of this study is arranged as follows. Firstly, we describe how we used the susceptible-exposed-infected- recovered-dormancy (SEIRD) model to simulate the impact that population migrations from Wuhan had on the number of infected patients in different cities. The following section describes how we empirically validated the theoretical inference based on data concerning population migrations and the number of confirmed cases. Based on theoretical and empirical research, we then describe China's experiences with regard to the prevention and control of the pandemic from the perspective of population migration.

Compared to studies by $\mathrm{Wu}$ et al. (2020), Zhao and Chen (2020), Tang et al. (2020), Anastassopoulou et al. (2020), Yang and Wang (2020), Chen et al. (2020ab), and Huang et al. (2020ab), this study has many unique features. First, we computed population migration into the SEIRD model and conducted a simulation analysis of the impact that migrating people, regarded as latently infected populations, had on the number of confirmed cases. Second, contrary to existing numerical simulation work, this study used statistical data to empirically validate the aforementioned theoretical inference. Finally, contrary to existing policy simulation and empirical research work, we introduced China's measures to prevent and control the spread of the pandemic, based on the variable of population migration. This article's research contributions are as follows. First, it enriches and expands on simulation and empirical research regarding the impact that population migration has on the COVID-19 pandemic. It offers an evaluation and early warning mechanism based on the variable of population migration, to help with the prevention and control of similar public health emergencies in the future. Second, its introduction to China's pandemic prevention and control measures, based on the variable of population migrations, is beneficial to effectively curb the global pandemic.

\section{Theoretical analysis}

\section{SEIRD model}

Considering that COVID-19 has an incubation period, we inferred that five states described the population. $S_{t}, E_{t}, I_{t}, R_{t}$, and $D_{t}$ represent the strength of susceptible populations, strength of exposed (latently infected) populations, strength of infected populations, strength of recovered populations, and size of dead populations at time $t$, respectively. The constant $N$ represents the total size of populations in the system, and the model assumes that $N \equiv S_{t}+R_{t}+E_{t}+I_{t}+D_{t}$.

It is assumed that latently infected and infected individuals came into contact with an average of $m_{1}$ and $m_{2}$ persons per day respectively, and that the infectiousness of latently infected and infected individuals is $\beta_{1}$ and $\beta_{2}$ respectively. The number of people that each latently infected and infected 
individual can infect are $m_{1} \beta_{1} E S / N$ and $m_{2} \beta_{2} I S / N$ respectively. Thus, the rate of change in the number of susceptible populations is as follows:

$\frac{d S}{d t}=-m_{1} \beta_{1} E S / N-m_{2} \beta_{2} I S / N$

Assuming that latently infected individuals become infected individuals at a probability of $\alpha$ each day, the rate of change in the number of latently infected individuals would be written as:

$\frac{d E}{d t}=m_{1} \beta_{1} E S / N+m_{2} \beta_{2} I S / N-\alpha E$

Assuming that infected individuals recover or die at the respective probabilities of $\gamma_{1}$ and $\gamma_{2}$ per day, the rate of change in the number of infected populations, number of recovered populations, and number of dead populations would respectively be:

$\frac{d I}{d t}=\alpha E-\gamma_{1} I-\gamma_{2} I$

$\frac{d R}{d t}=\gamma_{1} I$

$\frac{d D}{d t}=\gamma_{2} I$

To summarize, the SEIRD model is as follows:

$$
\left\{\begin{array}{l}
\frac{d S}{d t}=-m_{1} \beta_{1} E S / N-\frac{m_{2} \beta_{2} I S}{N} \\
\frac{d E}{d t}=m_{1} \beta_{1} E S / N+m_{2} \beta_{2} I S / N-\alpha E \\
\frac{d I}{d t}=\alpha E-\gamma_{1} I-\gamma_{2} I \\
\frac{d R}{d t}=\gamma_{1} I \\
\frac{d D}{d t}=\gamma_{2} I
\end{array}\right.
$$

\section{Initial parameter settings}

Following the example of Geng et al. (2020), we used the setting $\beta_{1}=\beta_{2}=0.045$ and considered that the setting $\alpha=1 /$ 7. According to COVID-19 data published by the National Health Commission of the People's Republic of China, the recovery ratio and mortality ratio fluctuated between 0.049 0.085 and $0.0009-0.0015$ respectively. The means of the intervals were taken, yielding the values $\gamma_{1}=0.069$ and $\gamma_{2}=$ 0.00115 . Assuming that the initial times for $I_{t}, R_{t}$, and $D_{t}$ are all $0, N=E_{0}+S_{0}=1$. Taking into account the difficulty of solving closed-form expressions, we have directly provided the results of the data simulation.

\section{Theoretical analysis of the population migration effect}

The size of populations migrating from Wuhan to each city primarily impacts E0 - the initial number of latently infected people in the cities. With this in mind, we summed up our analysis of the impact that the strength of populations, migrating from Wuhan, had on the number of infected cases. Notably, the analysis of the impact that E0, the number of initial latently infected cases, had on I $(\mathrm{t})$, the number of infected cases, was established. Considering that the number of infected cases is dynamic and that the number of newly infected cases follows an inverted U-shape trajectory, we hereby present the impact of the number of initial latently infected cases on the peak number of infected cases.

To ensure the robustness of the conclusion, the simulation was done under two conditions: frequent and infrequent contact among the public. When contact was frequent, $m_{1}=5$, and $m_{2}=3$; when contact was infrequent, $m_{1}=3$, and $m_{2}=1$. Figure 1 illustrates the specific results of the simulation. The vertical axis reflects the peak number of infected persons, while the horizontal axis denotes the proportion of initial populations migrating from Wuhan to various cities out of these cities' populations.

Figure 1 shows that regardless of whether a state of frequent or infrequent contact was instated among the public, the larger the population migrating from Wuhan to a city, the larger the initial latently infected population, and the higher the peak number of infected cases. Nevertheless, when members of the public were in frequent contact with one another, the peak number of infected cases was higher. Overall, based on theoretical analysis, we discovered the following: the larger the population migrating from Wuhan to each city in the initial period, the larger the infected population in each city in the later period. To validate the above proposition from an

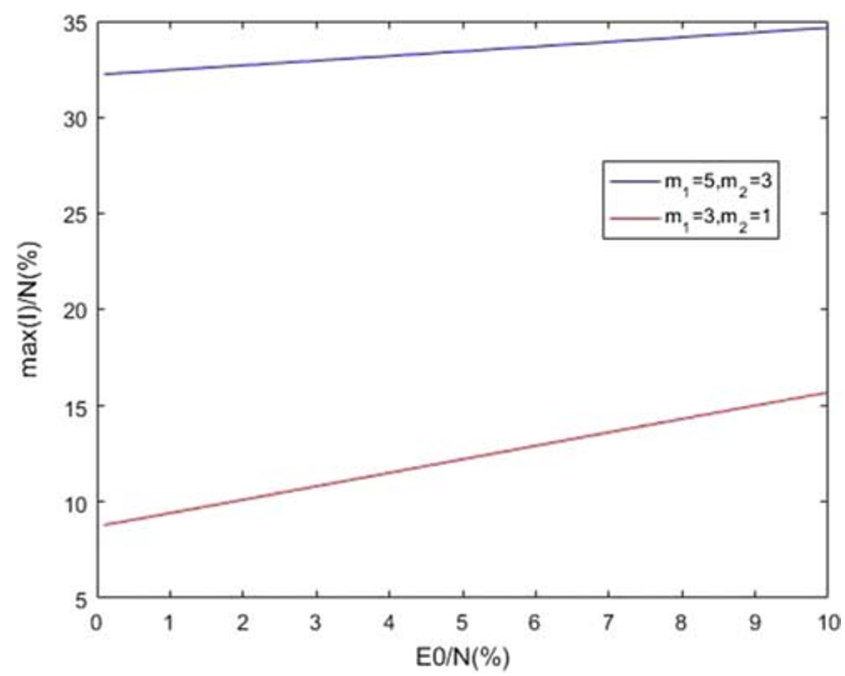

Fig. 1 Impact of population migration from the pandemic region on the number of infected cases 
empirical perspective, we analyzed the empirical relationship between the populations migrating from Wuhan to each city and the number of infected cases in each city.

\section{Empirical analysis}

\section{Quantitative model settings}

Limited by the data on populations migrating to various cities from Wuhan before Chinese New Year and till Wuhan's lockdown on January 23, we selected confirmed cases from the top 100 destinations for population migrations from Wuhan, beginning January $10-$ February 24 , as the outcome variable. Based on our theoretical analysis, we selected the number of people migrating from Wuhan to each city, and the resident population and population density in each city as the explanatory variables. To preserve the original mode of data, we selected the migration rate - the proportion of population migrating to each city from Wuhan out of the total migrant population from Wuhan - as the primary explanatory variable. While taking into account the dynamism of pandemic data, the number of confirmed cases was duly noted on 18th June 2020.

Given that the COVID-19 pandemic is exogenous, we used the ordinary least squares method for regression. The empirical model's settings are as follows:

Ninfected $_{i t}=\alpha+\beta$ Rmigration $_{i t}+\delta Z_{i t}+\varepsilon_{i t}$

In formula (7), Ninfected $_{i t}$ is the explained variable, highlighting the number of confirmed cases in the $i^{\text {th }}$ city at time $t$. is the main explanatory variable in this study, signifying the proportion of population migrating to the designated city from Wuhan out of the total population migrating from Wuhan, from January 10 to February 24, 2020. $Z_{i t}=\left(P_{i t}, P D_{i t}\right)$ represents the size of the resident population and population density, which affects the number of confirmed cases, and $\varepsilon_{\text {it }}$ represents other variables that impact the number of confirmed cases.

The number of confirmed cases in each city and the population migrating from Wuhan to each city were taken from Baidu's real-time dynamic pandemic monitoring system. Each city's resident population and geographical area were taken from the 2018 statistical yearbooks covering prefecture-level cities in China. Variables, such as the proportion of population migrating from Wuhan to each city and population density, were calculated using the aforementioned variables. Each variable's definition and descriptive statistics are shown in Table 1.

Table 1 affirms that apart from Wuhan, the average number of confirmed cases in each city was 276 . The region with the least cases was Heze, Shandong (18 cases), and the region with the most cases was Xiaogan, Hubei (3518 cases). Populations that headed for the top 100 destinations from Wuhan made up $91.58 \%$ of the total target population. Among them, the destination that made up the highest proportion $(13.8 \%)$ was Xiaogan, Hubei, while the destination that made up the lowest proportion (0.09\%) was Cangzhou, Hebei. In terms of total populations, Chongqing had the highest population (30.75 million), while Sanya, Hainan, had the lowest population ( 0.59 million). In terms of population density, Shenzhen had the highest density with 6202 persons per square kilometre, while Ankang, Shaanxi, had the lowest density with 113 persons per square kilometre.

\section{Impact of population migration on the number of confirmed cases}

To empirically validate the impact that the migrating populations from Wuhan have on the number of confirmed cases in each city, we provide four regression models. Model 1 applies only to the resident population in each city; Model 2 applies only to populations migrating from Wuhan to each city; Model 3 adds resident population to the foundation of Model 2; and Model 4 applies to the product term of migration rate and resident population, as well as population density. Table 2 outlines the results of the regression analysis.

Model 1 indicates that the coefficient for resident populations is non-significant, suggesting that cities without any source of infection would not have infected cases. Model 2 indicates that the larger the population migrating from Wuhan to each city, the greater the number of infected cases expected in each city. Particularly, with each increase of 1 percentage point in the proportion of populations migrating from Wuhan, the number of confirmed cases increased by 241. Given the fact that approximately 5 million persons migrated out of Wuhan during the Chinese New Year holidays, by deduction, each migration of 1000 individuals from Wuhan to a given region increases the number of confirmed cases within that region by 4.82 persons. Considering that the infection rate among Wuhan's resident population is $3.5 \%$, a single increase of a confirmed case from Wuhan increases the number of infected cases in a region by 0.14 persons.

Model 4 shows a positive and significant cross-correlation coefficient between the resident population and migration rate, which validates the theoretical inference by demonstrating that contact between infected and susceptible populations increases the number of infected cases. Model 4 also confirms that population density's impact on the number of confirmed cases was non-significant, which suggests that the key to suppressing the number of confirmed cases is to improve the public's pandemic literacy and response to a national call through active engagements in isolation. In summary, it is imperative to lower the number of infected cases by reducing 
Table 1 Statistical descriptions of variables

\begin{tabular}{|c|c|c|c|c|c|c|}
\hline Variable & Variable definition & $\begin{array}{l}\text { Sample } \\
\text { size }\end{array}$ & Mean & SD & Min & Max \\
\hline$N$ infected & $\begin{array}{l}\text { Number of confirmed cases; unit: } \\
\text { persons }\end{array}$ & 100 & 276 & 532 & 18 & 3518 \\
\hline Rmigration & $\begin{array}{l}\text { Migration rate; population } \\
\text { migrating from Wuhan to each } \\
\text { city/total population migrating } \\
\text { from Wuhan; unit: } \%\end{array}$ & 100 & 0.916 & 2.136 & 0.09 & 13.8 \\
\hline$P$ & $\begin{array}{l}\text { Total population; unit: } 10 \text { thousand } \\
\text { persons }\end{array}$ & 100 & 679.448 & 453.167 & 59.22 & 3075.16 \\
\hline PD & $\begin{array}{l}\text { Population density; resident } \\
\text { population/geographical area; } \\
\text { unit: persons }\end{array}$ & 100 & 729.682 & 797.805 & 113.762 & 6202.128 \\
\hline
\end{tabular}

$\mathrm{SD}=$ standard deviation the contact between infected and susceptible populations, as well as minimizing post-contact infectious agents.

\section{China's pandemic prevention and control based on population migration}

\section{Charting the direction of population migration and regions of focus for prevention and control}

China was able to prevent and control the spread of the pandemic based on the variable of population migration through the successful implementation of a multipronged strategy. First, specific population migration trends were obtained from Wuhan. The government collaborated with communication companies, such as China Mobile, technological companies like Baidu, and the Ministry of Communications, and was able to deduce the trajectory of population migration from Wuhan using the Global Positioning System (GPS) linked to mobile phone signals, Baidu Maps' street-navigation platform, and a real-name registration system linked with transportation. Next, regions necessitating precautionary measures were charted based on the population migration trends observed from Wuhan. As an example, through data obtained from
Baidu in a timely fashion, we discovered that Wuhan's populations migrated primarily to other prefecture-level cities in Hubei, such as Xiaogan, Huanggang, and Jingzhou. At the same time, we found that these regions were among prefecture-level cities with larger numbers of infected cases other than Wuhan, as shown in Table 3. Amid the peak of the pandemic, these prefecture-level cities should promptly activate emergency response policies, and fully equip themselves with human, financial, and material resources. Next, presumptive populations were identified within regions of focus. Through the aforementioned platforms and communities, the activity records of all presumptive personnel from a pandemic region were identified and tracked. Additionally, these cases were promptly reported to the pandemic prevention and control departments, and were isolated and issued notices on time. Such a strategy reduced contact between presumptive and confirmed populations, successfully preventing the pandemic from spreading.

\section{Intercepting population migration and post- migration population contact}

Amid a rapidly spreading pandemic, China successfully intercepted population migration and post-migration contact
Table 2 Impact of migration rate from Wuhan on the number of confirmed cases

\begin{tabular}{lllll}
\hline & Model 1 & Model 2 & Model 3 & Model 4 \\
\hline$P$ & $-0.00977(0.0906)$ & & $0.134^{* * * *}(0.0401)$ & \\
$R$ migration & & $240.9^{* * *}(10.52)$ & $244.4^{* * *}(11.11)$ & \\
PD & & & $-0.00729(0.0176)$ \\
$P * R$ migration & & & & $0.397^{* * * *}(0.0497)$ \\
${ }_{\text {cons }}$ & $282.8^{* * *}(96.19)$ & $55.62^{* * *}(12.71)$ & $-38.36(27.99)$ & $81.93^{* * * *}(28.75)$ \\
$N$ & 100 & 100 & 100 & 100 \\
$R^{2}$ & 0.000 & 0.933 & 0.945 & 0.791 \\
\hline
\end{tabular}

Note: $* * *, * *$, and $*$ respectively indicate levels of significance above $1 \%, 5 \%$, and $10 \%$; values within brackets are standard errors 
Table 3 The proportion of populations migrating from Wuhan out of the total number of confirmed cases in each city

\begin{tabular}{|c|c|c|c|c|}
\hline Ranking & Region & $\begin{array}{l}\text { Number of } \\
\text { confirmed cases }\end{array}$ & Region & $\begin{array}{l}\text { The proportion of the population that } \\
\text { migrated from Wuhan }(\%)\end{array}$ \\
\hline 1 & Xiaogan & 3518 & Xiaogan & 13.80 \\
\hline 2 & Huanggang & 2907 & Huanggang & 13.04 \\
\hline 3 & Jingzhou & 1580 & Jingzhou & 6.54 \\
\hline 4 & Ezhou & 1394 & Xianning & 5.01 \\
\hline 5 & Suizhou & 1307 & Ezhou & 3.97 \\
\hline 6 & Xiangyang & 1175 & Xiangyang & 3.93 \\
\hline 7 & Huangshi & 1015 & Huangshi & 3.77 \\
\hline 8 & Yichang & 931 & Jingmen & 3.30 \\
\hline 9 & Jingmeng & 928 & Suizhou & 3.21 \\
\hline 10 & Xianning & 836 & Xiantao & 2.97 \\
\hline 11 & Beijing & 752 & Yichang & 2.81 \\
\hline 12 & Shanghai & 697 & Tianmen & 2.08 \\
\hline 13 & Shiyan & 672 & Shiyan & 1.86 \\
\hline 14 & Chongqing & 582 & Enshi & 1.81 \\
\hline 15 & Xiantao & 575 & Xinyang & 1.49 \\
\hline 16 & Wenzhou & 504 & Chongqing & 1.27 \\
\hline 17 & Tianmeng & 496 & Qianjiang & 1.14 \\
\hline 18 & Shenzhen & 423 & Changsha & 1.02 \\
\hline 19 & Guangzhou & 377 & Beijing & 0.86 \\
\hline 20 & Xinyang & 274 & Nanyang & 0.69 \\
\hline 21 & Harbin & 264 & Shanghai & 0.66 \\
\hline 22 & Enshi & 252 & Zhumadian & 0.66 \\
\hline 23 & Changsha & 242 & Zhenzhou & 0.59 \\
\hline 24 & Nanchang & 230 & Jiujiang & 0.52 \\
\hline 25 & Qianjiang & 198 & Yueyang & 0.52 \\
\hline 26 & Tianjin & 197 & Shenzhen & 0.50 \\
\hline 27 & Hangzhou & 181 & Guangzhou & 0.50 \\
\hline 28 & Hefei & 174 & Nanchang & 0.48 \\
\hline 29 & Zhengzhou & 157 & Chengdu & 0.46 \\
\hline 30 & Ningbo & 157 & Anqing & 0.45 \\
\hline
\end{tabular}

among populations. First, traffic from Wuhan had to be promptly cut off to all cities, and regions which the disease had migrated to severed all traffic connections with the source. On January 23, Wuhan City's COVID-19 Pandemic Prevention and Control Headquarters announced that the operations of Wuhan's regular and long-distance buses, subways, and ferries were suspended. Routes leaving Wuhan, including airports and train stations, were temporarily closed as well. On January 25, the operations of four high-speed rail trains from Zhengzhou to Wuhan were suspended. Additionally, traffic and commuting within the restricted region was banned, to reduce interactions among individuals. For example, Wuhan City's COVID-19 Epidemic Prevention and Control Headquarters announced that starting from January 26 , a rule prohibiting motor vehicle activity took effect in the downtown region. Following this, Huanggang, Hubei announced a suspension of all bus operations in urban areas and a universal cancellation of gatherings and group dining activities. Again, lockdown management was enforced to restrict residents from being active in that community. News and official media promotions were heightened, reminding residents to wear masks, avoid going outside, or attend gatherings. Community surveillance work was employed, and residents who did not comply with warnings and arrangements were publicly exposed and subjected to legal prosecutions.

\section{Concluding remarks}

Under the current international pandemic, which worsens by the day, population migration has a critical effect on the pandemic's spread. The surge of the disease was effectively prevented and controlled in China, and the study deciphers 
the migration patterns in light of the SEIRD model to track the spread of COVID-19. Through our simulation of the mechanism of influence that population migration had on the number of confirmed cases in each region, we found that by increasing the number of latently infected cases in each city at the initial stage, the strength of populations migrating from Wuhan to each city increased the number of confirmed cases in each city. Reducing contact between susceptible populations and infected populations could effectively lower the number of infected cases. Our empirical findings further validated the theoretical inference that 'the larger the population migrating from Wuhan to each city, the greater the population of confirmed cases in each city'.

Based on our theoretical and empirical analysis of the impact that population migration in China had on the number of confirmed cases, how did China prevent and control the spread of the pandemic? First, the directions of population migration from the source of the pandemic were confirmed, and regions of focus for prevention and control based on the variable of the population were highlighted. Next, after identifying regions of focus, contact between susceptible and infected populations within the regions was effectively intercepted. This study's research contributions lie in its enrichment of theoretical and empirical research on the impact that population migration has on a pandemic's spread. Furthermore, it provides an aspect of China's experience regarding the prevention and control of the current pandemic and encompasses lessons for early warning, prevention, and control of similar public health emergencies.

Funding This study was funded by the Ministry of Education of Humanities and Social Science project (Grant number: 19YJC790167; 20YJC790187).

\section{Compliance with ethical standards}

Conflict of interest The authors declare that they have no conflict of interest.

Ethical approval and informed consent Not applicable.

\section{References}

Anastassopoulou C, Russo L, Tsakris A, Siettos C (2020) Data-based analysis, modelling and forecasting of the COVID-19 outbreak. PLoS ONE 15(3):e0230405. https://doi.org/10.1371/journal.pone. 0230405

Chen TM, Rui J, Wang QP, Zhao ZY, Cui JA, Yin L (2020a) A mathematical model for simulating the phase-based transmissibility of a novel coronavirus. Infect Dis Poverty 9(1):24-24. https://doi.org/10. 1186/s40249-020-00640-3

Chen S, Yang J, Yang W, Wang C, Bärnighausen T (2020b) COVID-19 control in China during mass population movements at new year.
Lancet 395(10226):764-766. https://doi.org/10.1016/S01406736(20)30421-9

Deng SQ, Peng HJ (2020) Characteristics of and public health responses to the coronavirus disease 2019 outbreak in China. J Clin Med 9(2): 575

Geng H, Xu AD, Wang XY (2020) Analysis of the role of current prevention and control measures in the epidemic of new coronavirus based on SEIR model. J Jinan Univ (Nat Sci Med Ed) 41(2):1-7

Gong F, Xiong Y, Xiao J, Lin L, Liu X, Wang D, Li X (2020) China's local governments are combating COVID-19 with unprecedented responses - from a Wenzhou governance perspective. Front Med China 14(2):220-224

Guan W, Ni Z, Hu Y et al (2020) Clinical characteristics of coronavirus disease 2019 in China. N Engl J Med 382:1708-1720. https://doi. org/10.1056/NEJMoa2002032

Huang R, Liu M, Ding Y (2020a) Spatial-temporal distribution of COVID-19 in China and its prediction: a data-driven modeling analysis. J Infect Dev Countries 14(3):246-253

Huang C, Wang Y, Li X et al (2020b) Clinical features of patients infected with 2019 novel coronavirus in Wuhan, China. Lancet 395(10223):497-506. https://doi.org/10.1016/S0140-6736(20) 30183-5

Kucharski AJ, Russell TW, Diamond C et al (2020) Early dynamics of transmission and control of COVID-19: a mathematical modelling study. Lancet Infect Dis 134(5):543-545. https://doi.org/10.1016/ S1473-3099(20)30161-4

Li Q, Guan X, Wu P et al (2020) Early transmission dynamics in Wuhan, China, of novel coronavirus-infected pneumonia. N Engl J Med 382(13):1199-1207. https://doi.org/10.1056/NEJMoa2001316

Prem K, Liu Y, Russell TW et al (2020) The effect of control strategies to reduce social mixing on outcomes of the COVID-19 epidemic in Wuhan, China: a modelling study. Lancet Public Health 5(5):e261e270. https://doi.org/10.1016/S2468-2667(20)30073-6

Tang B, Wang X, Li Q (2020) Estimation of the transmission risk of the 2019-nCoV and its implication for public health interventions. J Clin Med 9(2):462. https://doi.org/10.3390/jcm9020462

Tian H, Liu Y, Li Y et al (2020) An investigation of transmission control measures during the first 50 days of the COVID-19 epidemic in China. Science 368(6491):eabb6105. https://doi.org/10.1126/ science.abb6105

Wang G, Jin X (2020) The progress of 2019 novel coronavirus event in China. J Med Virol 92(5):468-472. https://doi.org/10.1002/jmv. 25705

Wu JT, Leung K, Leung GM (2020) Nowcasting and forecasting the potential domestic and international spread of the 2019-nCoV outbreak originating in Wuhan, China: a modelling study. Lancet 395(10225):689-697. https://doi.org/10.1016/S0140-6736(20) 30260-9

Xu J, Chen Y, Chen H, Cao B (2020) 2019 novel coronavirus outbreak: a quiz or final exam? Front Med China 14(2):225-228. https://doi. org/10.1007/s11684-020-0753-1

Yang CY, Wang J (2020) A mathematical model for the novel coronavirus epidemic in Wuhan, China. Math Biosci Eng 17(3):2708-2724

Zhang H (2020) Early lessons from the frontline of the 2019-nCoV outbreak. Lancet 395(10225):687

Zhang S, Wang Z, Chang R et al (2020) COVID-19 containment: China provides important lessons for global response. Front Med China 15. https://doi.org/10.1007/s11684-020-0766-9

Zhao SL, Chen H (2020) Modeling the epidemic dynamics and controlof COVID-19 outbreak in China. Quantitative Biol 8(1):11-19

Publisher's note Springer Nature remains neutral with regard to jurisdictional claims in published maps and institutional affiliations. 